\title{
Experimental and Theoretical Study on the Ethane and Acetylene Formation from Electron Irradiation of Methane Ices
}

\author{
Marianna Barberio $^{1 *}$, Roberta Vasta ${ }^{1,2}$, Pasquale Barone $^{1}$, Giulio Manicò ${ }^{3}$, Fang $\mathrm{Xu}^{1}$ \\ ${ }^{1}$ Physics Department of Calabria Univeristy, Rende, Italy; ${ }^{2}$ National Research Council-CNR, Magna Graecia University, Catanzaro, \\ Italy; ${ }^{3}$ Astronomy and Physics Department, University of Catania, Catania, Italy. \\ Email: ${ }^{*}$ mmarianna.barberio@fis.unical.it
}

Received October $3^{\text {rd }}, 2012$; revised November $9^{\text {th }}, 2012$; accepted November $21^{\text {st }}, 2012$

\begin{abstract}
In this work we present an experimental and theoretical study on the formation of ethane and acetylene from solid methane condensed at $20 \mathrm{~K}$ and irradiated with a $500-3000 \mathrm{eV}$ electron beam. The experiments were monitored with Thermal Desorption Spectroscopy. We observe that the electron irradiation induced a dehydrogenation of methane and a consequent formation of $\mathrm{CH}_{\mathrm{x}}(\mathrm{x}=1,2,3)$ fragments. Furthermore, in the solid during irradiation, a simple recombination reaction in the solid between two adjacent $\mathrm{CH}_{\mathrm{x}}$ molecules may form $\mathrm{HC} \equiv \mathrm{CH}, \mathrm{H}_{2} \mathrm{C}=\mathrm{CH}_{2}$, and $\mathrm{H}_{3} \mathrm{C}-\mathrm{CH}_{3}$ with a triple, double, and single carbon-carbon bond, respectively. The formed amount of ethane and acetylene increases with irradiation time and reaches a saturation value.
\end{abstract}

Keywords: Solid Ices; Ethane Formation; Acetylene Formation; Electron Irradiation

\section{Introduction}

In the past decades, self assembled monolayers and multilayers of different materials have attracted growing attention in many areas of practical applications such as protein microsensors, electrochemical interfaces, microelectronics development and surface science investigations [1].

In particular the study of interaction between these thin films and charged and energetic particles has been developed for its implications in astrophysics and in spatial device applications [1-3]. In this context it is very important to obtain information about the formation of macromolecules due to the interaction between the interstellar medium and the charged and energetic particles of cosmic radiation.

The interstellar medium (ISM) is composed of gas for $99 \%$ and constitutes about $10 \%$ of the mass of our Galaxy. Hydrogen and helium represent respectively $93.38 \%$ and $6.49 \%$ of ISM chemical composition, while oxygen, carbon and nitrogen contribute only with $0.11 \%$ $(\mathrm{O}: \mathrm{C}: \mathrm{N}=6: 3: 1,[4])$.

The ISM is not distributed uniformly in the space between stars but settles in the galactic plane, where it "condenses" in large-scale structures as for example the dense interstellar clouds. In these environments a rich

*Corresponding author. chemistry takes place, actually around 140 molecular species have been identified.

Atoms, molecules, and radicals in the gas phase can condense on the surface of dust grains forming an icy mantle with a thickness of about $0.1 \mu \mathrm{m}$. The presence of ices is confirmed by IR spectroscopy of obscured stellar sources. Icy mantles are constituted by a solid mixture containing $\mathrm{H}_{2} \mathrm{O}, \mathrm{CO}, \mathrm{CH}_{4}$ and $\mathrm{NH}_{3}$ [5,6]. Ices in the molecular clouds and solar system bodies are subjected to the action of cosmic rays, UV photons and energetic particles. New complex organic species may form during interactions with UV radiations or energetic ions [7], while ionization may occur if the ices are irradiated with cosmic rays. Though the icy mantles are rarely under energetic electron bombardment, the electrons emitted in ionization processes can be energetic enough to further ionize or excite surrounding molecules creating photons, electrons, ions, radicals, and roto-vibronically excited species [8]. In some cases, a simple local warming up may also facilitate some reactions [9].

Therefore it is essential to understand the effects of electron bombardment on the chemical composition of the ices [10]. Several systematic studies have been reported in the literature concerning the interactions of electrons with various astronomically relevant molecular ices as $\mathrm{H}_{2} \mathrm{O}, \mathrm{NH}_{3}, \mathrm{CH}_{4}$ etc. and their mixtures by em- 
ploying infrared spectroscopy and thermal desorption (TD) techniques [2,10-12].

In particular, studies of chemical composition of comets $[11,12]$ suggest that the formation of hydrocarbons, ethane and acetylene mostly, is originated from the irradiation of methane within interstellar ices. In this framework the study of production of new hydrocarbons formed from the irradiation of methane assumes particular importance. Recently Bennet et al. [3] performed the irradiation of methane ices at $10 \mathrm{~K}$ with energetic electrons $(5 \mathrm{keV})$ to mimic the energy transfer process that occur in the track of the trajectories of $\mathrm{MeV}$ cosmic ray particles. Their experiment, monitored via FTIR spectroscopy, comes to conclusions that the primary effects of irradiation is the formations of methyl radical $\mathrm{CH}_{3}$ and that subsequently in the matrix two neighboring methyl radicals can recombine to form ethane molecule $\left(\mathrm{C}_{2} \mathrm{H}_{6}\right)$, finally the irradiations of these new molecules generated ethylene, ethyl radical, vinyl radical and acetylene as degradation products. However, in this study the irradiation time is limited to 50 minutes and the ethane production is linearly increasing without showing any sign of turn up or stabilization on a saturation value.

In this work we present an experimental and theoretical investigation on $500-3000 \mathrm{eV}$ electron induced chemical reaction in solid methane at $20 \mathrm{~K}$. Our study confirms and improves that one of Bennet et al. Indeed we obtain as Bennet [3] that the primary effects of irradiations is the methyl radical formations, followed by the formations of ethane and of other hydrocarbons. Moreover we demonstrated, for first time, that this process is possible also for low energy electrons (in the range 500 $3000 \mathrm{eV}$ ) and that for very large irradiation time (about 250 minutes) the ethane production leads a saturation value.

\section{Experiments}

The experiments were conducted in an ultra high vacuum chamber (base pressure in the low $10^{-10}$ mbar range) evacuated with a dry turbo pump. A computer controlled quadruple mass spectrometer was used to monitor various gas species released during the thermal desorption processes. For each of them, a mass scan of about 40 data points was taken and the integrated area was used as the signal intensity. To better detect all desorbed gases, an automatically adjusted and differently set sensibility scale was used for each mass.

Methane ice was formed by condensing $\mathrm{CH}_{4}$ onto the stainless steel cold head surface $\left(7 \mathrm{~cm}^{2}\right)$ of a close cycled He refrigerator by introducing $\mathrm{CH}_{4}$ gas (purity of $99.999 \%$ ) in the chamber through a precision leak valve. Background dosing allowed formation of a uniform film while use of a $2 \mathrm{~mm}$ diameter $\mathrm{Cu}$ tube with its end reproducibly positioned at $<1 \mathrm{~mm}$ away from the cold surface enabled the growth of a column-like bulk film. In the latter case, the gas pressure in the tube and the dosing time were kept constant. The sample temperature was monitored with a calibrated $\mathrm{Au} / 0.7 \%$ Fe-chromel thermocouple and the adsorption temperature was $20 \pm 2 \mathrm{~K}$. Sample heating was achieved by simply turning off the refrigerator compressor. In the temperature range of our interest (40 - 120 $\mathrm{K})$ the heating rate was nearly constant $(3 \mathrm{~K} / \mathrm{min})$. Quadrupole mass spectra and the sample temperature recording were synchronized.

We observe that, for background dosing, two distinct desorption peaks were observed for as deposited $\mathrm{CH}_{4}$ films with a temperature difference of $10 \mathrm{~K}$ and their intensity ratio remained constant as the amount of the adsorbed $\mathrm{CH}_{4}$ varied. This was due to adsorption on two different portions of the cold head, as checked with a set of thermocouples positioned on various parts of the second stage of the refrigerator. The desorption features reported in this paper are relative to those coming from the coldest area which were subjected to electron irradiation.

In studying the effects of interactions with the $\mathrm{CH}_{4}$ ice, the electron beam with energy of $200-3000 \mathrm{eV}$ was totally defocalized with a spot size similar to the sample surface area to avoid the local heating. The beam current was in the order of $\mu \mathrm{A}$ and the bombardment time ranged from a few minutes up to 250 minutes.

Tiny traces of $\mathrm{H}_{2} \mathrm{O}, \mathrm{N}_{2}$ and $\mathrm{O}_{2}$ were detected during desorption but they were absent if the chamber was directly filled with gases suggesting that they were due to condensation of the chamber residual contaminant species.

\section{Results and Discussions}

\subsection{Experimental Results}

In Figure 1 we present four typical mass spectra taken at temperatures of $T=47 \mathrm{~K}$ and $T=62 \mathrm{~K}$ during thermal desorption of a thin solid methane film deposited with $350 \mathrm{~L}$ background dosing $1 \mathrm{~L}=1 \times 10^{-6}$ Torr $\cdot$ sec. Spectra (a) and (b) are for the sample irradiated with $2000 \mathrm{eV}$ electrons while spectra (c) and (d) are relative to an as deposited film. For the purpose of comparison we also show the mass spectra taken by filling the chamber at $1 \times$ $10^{-6}$ mbar with room temperature $\mathrm{CH}_{4}, \mathrm{C}_{2} \mathrm{H}_{6}$, and $\mathrm{H}_{2}$ gases (curves (e)-(g), respectively).

We first note that the quadrupole filament (operating at $60 \mathrm{~V}[13,14])$ produced a great amount and variety of fragments for methane and ethane. This certainly overshadowed the effects of electron irradiation on solid $\mathrm{CH}_{4}$. Indeed, the intensity ratios of $\mathrm{CH}_{\mathrm{x}} / \mathrm{CH}_{4}$ and $\mathrm{C}_{2} \mathrm{H}_{\mathrm{x}} / \mathrm{C}_{2} \mathrm{H}_{6}$ are very similar for curves (a), (c) and (e) and for curves 


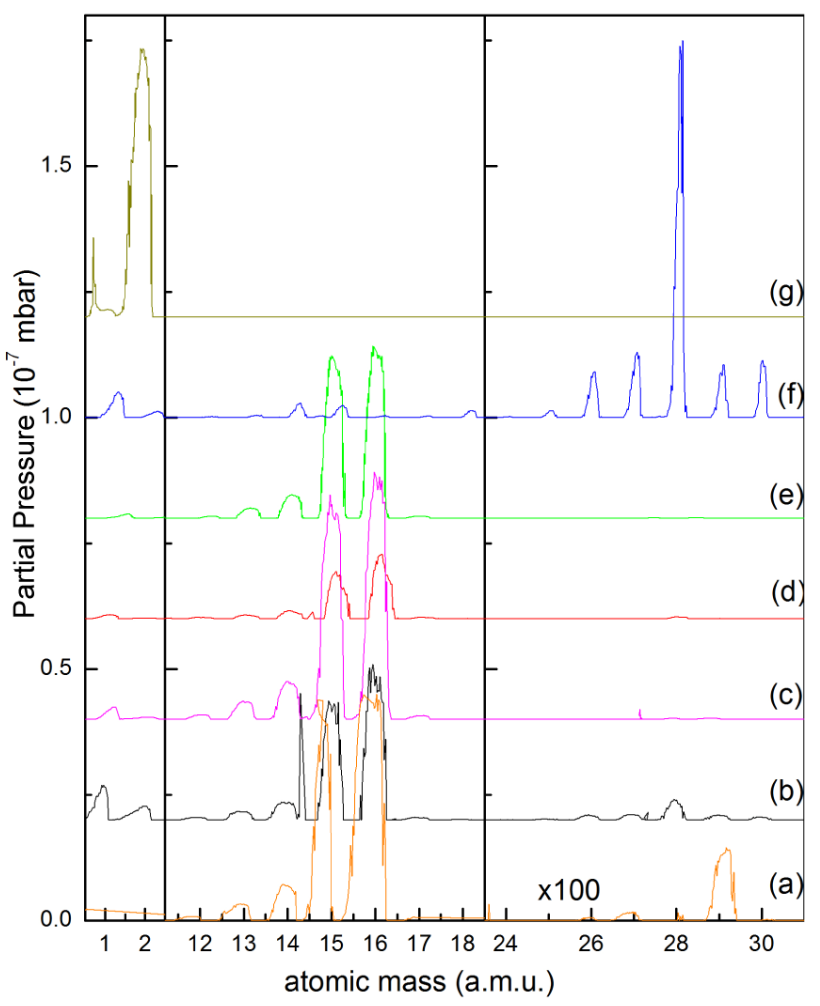

Figure 1. Quadruple mass spectra taken during thermal desorption of a $2 \mathrm{keV}$ electron bombarded thin $\mathrm{CH}_{4}$ film at $47 \mathrm{~K}$ (curve (a)) and $62 \mathrm{~K}$ (curve (b)) and for an as-deposited film (curves (c) and (d)). Also shown are the scans for background filled methane, ethane and hydrogen gases at $1 \times$ $10^{-6}$ mbar (curves (e)-(g), respectively). For the sake of clarity, all curves have been shifted.

(b), (d) and (f), respectively, clearly indicating that cracking due to the quadrupole filament is by far the most dominant mechanism for the observed dissociation of methane and ethane.

Thermal desorption curves of the relevant species for an electron bombarded sample are illustrated in Figure 2. The film was obtained by background dosing and its thickness was approximately $1350 \AA$ (or 350 layers) allowing the primary electrons of $2000 \mathrm{eV}$ to penetrate through. The electron flux was $\Phi=2 \times 10^{16}$ electrons $/ \mathrm{cm}^{2}$. The spectra can be gathered into three groups which appear separately in different temperature ranges. Methane and its derivates $\mathrm{CH}_{\mathrm{x}}$ are released at about $47 \mathrm{~K}$, atomic and molecular hydrogen signals are seen at $29 \mathrm{~K}$ and together with $\mathrm{CH}_{\mathrm{x}}$ at $47 \mathrm{~K}$, whereas the $\mathrm{C}_{2} \mathrm{H}_{\mathrm{x}}$ species desorb at higher temperatures with two wider features centered at 62 and $75 \mathrm{~K}$. We notice that for $T>50 \mathrm{~K}$, the TPD curve for $\mathrm{CH}_{4}$ is structureless though its intensity is much higher than those of $\mathrm{C}_{2} \mathrm{H}_{\mathrm{x}}$ species. For as deposited films no dicarbonic molecules were detected.

To further study the irradiation effects, we condensed a column-like bulk $\mathrm{CH}_{4}$ with relatively small quantity of methane gas avoiding the over pressure during desorp-

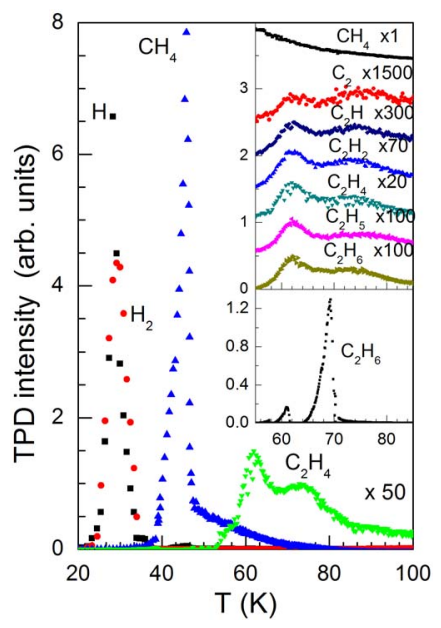

Figure 2. Thermal desorption traces of some relevant species for a background dosed methane film of $450 \mathrm{~L}$ after 60 min of $2 \mathrm{keV}$ electron bombardment at a current density of $880 \mu \mathrm{A} / \mathrm{cm}^{2}$. In the upper panel of the inset are shown the traces of $\mathrm{C}_{2} \mathrm{H}_{\mathrm{x}}$ while that illustrated in the lower panel is for an as deposited ethane film.

tion. The thickness was estimated to be a few tens of mm and quite reproducible as judged by the total desorbed $\mathrm{CH}_{4}$ signal. The monotonous increase of the amount of desorbed $\mathrm{C}_{2} \mathrm{H}_{6}$ plotted in Figure 3 as a function of the electron irradiation time clearly demonstrates the effects of electron stimulated reactions.

In discussing the electron induced dehydrogenation occurring in the solid methane matrix, we mention that the energy required for $\mathrm{CH}_{4}$ dissociation is only a few eV so that the impinging primary electrons and also seconddary electrons generated in the cascade could produce $\mathrm{CH}_{\mathrm{x}}$ fragments with $\mathrm{CH}_{3}$ being the most important one [Shirai et al. [15], Table 1]. Because of the dominant quadrupole cracking effects on desorbing methane molecules from a large portion of cold surfaces which were not subjected to electron bombardment, a direct detection of these $\mathrm{CH}_{\mathrm{x}}$ reaction products through thermal desorption spectra was difficult. However, these reactions could still be inferred from the observation of molecular hydrogen during electron irradiation. Indeed, turning on and off the electron beam with use of a mechanical shuttle resulted in a quite reproducible on-off behavior of the $\mathrm{H}_{2}$ signals.

In the solid, to the molecular dissociation may follow a recombination process, which is negligible in gas phase because of the large intermolecular distance. The most favored recombination reactions are those with very low binding enthalpies, as the formation of hydrogen molecules, acetylene, ethylene, and ethane. A simple recombination reaction between two adjacent $\mathrm{CH}_{\mathrm{x}}(\mathrm{x}=1,2,3)$ molecules may form $\mathrm{HC} \equiv \mathrm{CH}, \mathrm{H}_{2} \mathrm{C}=\mathrm{CH}_{2}$, and $\mathrm{H}_{3} \mathrm{C}-\mathrm{CH}_{3}$ with a triple, double, and single carbon-carbon bond, respectively. 


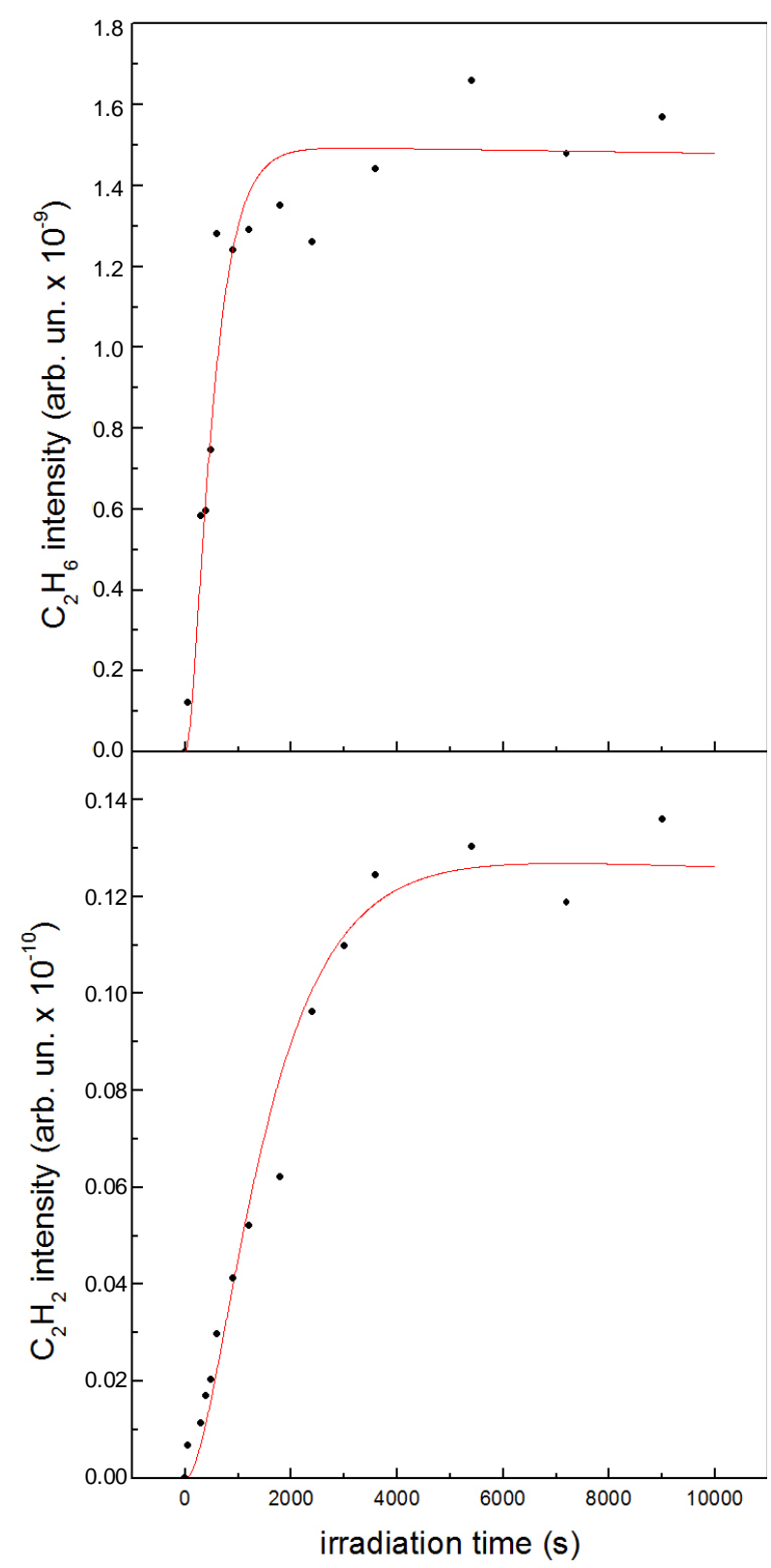

Figure 3. Total $\mathrm{C}_{2} \mathrm{H}_{6}$ (a) and $\mathrm{C}_{2} \mathrm{H}_{2}$ (b) intensity produced by $2 \mathrm{keV}$ electron bombardment on a thick solid $\mathrm{CH}_{4}$ film as a function of irradiation time. The continuous curve is a best fit by using a simple rate-equation based recombination model (see text).
The fact that all $\mathrm{C}_{2} \mathrm{H}_{\mathrm{x}}$ TPD curves have nearly the same line shape (upper panel of the inset of Figure 2) would strongly suggest an essentially common origin of these species. A comparison of the intensity ratios seen in the TPD curves between $\mathrm{C}_{2} \mathrm{H}_{\mathrm{x}}$ and $\mathrm{C}_{2} \mathrm{H}_{6}$ with those recorded for ethane gas (curve (g) of Figure 1) reveals that virtually all observed dicarbonic species could be attributed to the quadrupole filament cracking of desorbed ethane molecules except for acetylene. Indeed, in this later case, the detected intensity ratio is much higher than the cracking faction. This observation leads us to conclude that a substantial portion of $\mathrm{C}_{2} \mathrm{H}_{2}$ must have formed in the $\mathrm{CH}_{4}$ matrix upon electron irradiation.

A comparison with the TPD curve of a solid $\mathrm{C}_{2} \mathrm{H}_{6}$ film (lower panel of the inset in Figure 2) show that the binding environments of electron irradiation produced ethane are much more complex with two broad structures centered at about 62 and $73 \mathrm{~K}$, in contrast to the sharp desorption peak of bulk ethane at $69 \mathrm{~K}$.

\subsection{Theoretical Model}

We point out that the difference in the desorption temperatures for $\mathrm{CH}_{3}(\mathrm{CH})$ and $\mathrm{C}_{2} \mathrm{H}_{6}\left(\mathrm{C}_{2} \mathrm{H}_{2}\right)$ species unambiguously indicates that ethane (acetylene) molecules are formed in the solid during electron irradiation or during thermal annealing, but certainly not in gas phase during desorption.

To get some estimates on the process of $\mathrm{CH}_{4}$ dehydrogenation into $\mathrm{CH}_{3}$ and the subsequent $\mathrm{CH}_{3}$ recombination into $\mathrm{C}_{2} \mathrm{H}_{6}$, we first note that the probability that a molecule within the reaction depth $l$ gets hit by a $2 \mathrm{keV}$ electron in a second is $P_{1}=\frac{I}{1.6 \times 10^{-19} \rho l}$ where $I=8.8 \times 10^{-3} \mathrm{~A} \cdot \mathrm{m}^{-2}$ is the electron beam density and $\rho=$ $1.694 \times 10^{28} \mathrm{~m}^{-3}$ the site number density. The probability a methane molecule undergoes a collision with a primary electron is $P_{1} \cdot \theta_{\mathrm{CH}_{4}}$ with $\theta_{\mathrm{CH}_{4}}$ denoting the $\mathrm{CH}_{4}$ concentration: $\theta_{\mathrm{CH}_{4}}(t=0)=1$ and $\theta_{\mathrm{CH}_{4}}(t=\infty)=0$. In the case of background dosing as that shown in Figure 2, the film thickness $d=135 \mathrm{~nm}$ was smaller than the primary electron penetration length $\lambda$ [16-18] so that chemical reaction took place through the whole sample thickness:

Table 1. cross section $\left(\mathrm{cm}^{2}\right)$ vs electron energy for emission of product from $\mathrm{CH}_{4}$ electron irradiation.

\begin{tabular}{cccc}
\hline & $20 \mathrm{eV}$ (secondary electrons) & $60 \mathrm{eV}$ (quadrupole filament electrons) & $2000 \mathrm{eV}$ (electron beam energy) \\
\hline $\mathrm{CH}_{3}$ & $5 \times 10^{-18}$ & $2 \times 10^{-16}$ & $9 \times 10^{-17}$ \\
$\mathrm{CH}_{2}$ & $1 \times 10^{-18}$ & $2 \times 10^{-17}$ & $3 \times 10^{-18}$ \\
$\mathrm{CH}$ & $3.01 \times 10^{-19}$ & $2.2 \times 10^{-17}$ & $2.2 \times 10^{-18}$ \\
$\mathrm{C}$ & $1.03 \times 10^{-19}$ & $3.4 \times 10^{-18}$ & $3.34 \times 10^{-19}$ \\
$\mathrm{H}$ & $1.23 \times 10^{-19}$ & $2.437 \times 10^{-17}$ & $3.267 \times 10^{-18}$ \\
\hline
\end{tabular}


$l=d, P_{1}=2.45 \times 10^{-5}$. The dissociation probability for $\mathrm{CH}_{4}$ is $P_{2}=\frac{\sigma}{\pi r^{2}}=8.05 \times 10^{-2}$ with $\sigma=9.5 \times 10^{-17} \mathrm{~cm}^{2}$ being the cross section at $2 \mathrm{keV}$ (Table 1) and $r=1.95 \AA$ the molecular mean radius. Therefore, the probability that a $\mathrm{CH}_{4}$ molecule could be dissociated in a second is $P_{1} \cdot \theta_{\mathrm{CH}_{4}} \cdot P_{2}=1.97 \times 10^{-6} \theta_{\mathrm{CH}_{4}}$. Considering that a $2 \mathrm{keV}$ electron produces many secondary electrons, we may reasonable assume the total number of hits to be at least 50 with a similar s, then the real probability of dissociating a $\mathrm{CH}_{4}$ molecule in a second can be as high as $1 \times$ $10^{-4} \theta_{\mathrm{CH}_{4}}$. For $\theta_{\mathrm{CH}_{4}}=1$ the total probability of dehydrogenation in an hour is $P=36 \%$, thus as a first approximation we may take a simple average value of $\theta_{\mathrm{CH}_{4}}=\frac{1+(1-0.36)}{2}=0.82$ so that $P=30 \%$.

Experimentally, the intensity ratio between $\mathrm{C}_{2} \mathrm{H}_{6}$ (including its cracking fragments) and $\mathrm{CH}_{4}$ originating from electron bombarded region is about $1.8 \times 10^{-2}$, thus that between $\mathrm{CH}_{3}$ involved in the recombination and $\mathrm{CH}_{4}$ is $3.6 \times 10^{-2}$. This later value indicates that only about $10 \%$ of produced $\mathrm{CH}_{3}$ recombine to form $\mathrm{C}_{2} \mathrm{H}_{6}$.

To somehow quantitatively describe the kinetics of electron induced reactions occurring in the solid matrix (case of column-like thick film), we assume that the film thickness to be much larger than the electron penetration length and denote with $\theta_{\mathrm{CH}_{4}}$ the concentration of $\mathrm{CH}_{4}$ molecules. The rate equation for methane [2] can be written as

$$
\frac{\mathrm{d} \theta_{\mathrm{CH}_{4}}}{\mathrm{~d} t}=-c_{1} \theta_{\mathrm{CH}_{4}}+c_{2} \theta_{\mathrm{CH}_{3}} \cdot \theta_{H}
$$

where $c_{1}$ is the rate constant for reduction of $\mathrm{CH}_{4}$ into $\mathrm{CH}_{\mathrm{x}}(\mathrm{x}=0,1,2,3)$ and $c_{2}$ that for reformation from $\mathrm{CH}_{3}$ and $\mathrm{H}$. Because of the preferential formation of $\mathrm{H}_{2}$ and its instant release from the solid, we can reasonably assume $c_{2}=0$. Equation (1) then has a simple solution

$$
\theta_{\mathrm{CH}_{4}}(t)=\theta_{\mathrm{CH}_{4}}^{0} \mathrm{e}^{-c_{1} t}
$$

where $\theta_{\mathrm{CH}_{4}}^{0}$ denotes the initial density of $\mathrm{CH}_{4}$.

The rate equation for $\mathrm{CH}_{3}$ molecules [2] is given by

$$
\frac{\mathrm{d} \theta_{\mathrm{CH}_{3}}}{\mathrm{~d} t}=c_{3} \theta_{\mathrm{CH}_{4}}-c_{4} \theta_{\mathrm{CH}_{3}}=\theta_{\mathrm{CH}_{4}}^{0} c_{3} \mathrm{e}^{-c_{1} t}-c_{4} \theta_{\mathrm{CH}_{3}}
$$

where $c_{3}$ is the rate constant for dissociation of $\mathrm{CH}_{4}$ into $\mathrm{CH}_{3}$ and $c_{4}$ that for fragmentation of $\mathrm{CH}_{3}$ into smaller ones. The solution of (3) satisfying the initial condition $\theta_{\mathrm{CH}_{3}}(t=0)=0$ is:

$$
\theta_{\mathrm{CH}_{3}}(t)=\frac{c_{3}}{c_{4}-c_{1}} \theta_{\mathrm{CH}_{4}}^{0}\left(\mathrm{e}^{-c_{1} t}-\mathrm{e}^{-c_{4} t}\right)
$$

As the $\mathrm{CH}_{3}+\mathrm{CH}_{3} \rightarrow \mathrm{C}_{2} \mathrm{H}_{6}$ recombination is concerned, if it occurs at low temperature during electron irradiation, then its rate equation is given as

$$
\begin{aligned}
& \frac{\mathrm{d} \theta_{\mathrm{C}_{2} \mathrm{H}_{6}}}{\mathrm{~d} t}=c_{5} \theta_{\mathrm{CH}_{3}} \cdot \theta_{\mathrm{CH}_{3}}-c_{6} \theta_{\mathrm{C}_{2} \mathrm{H}_{6}} \\
= & \alpha\left(\mathrm{e}^{-2 c_{1} t}+\mathrm{e}^{-2 c_{4} t}-2 \mathrm{e}^{-\left(c_{1}+c_{4}\right) t}\right)-c_{6} \theta_{\mathrm{C}_{2} \mathrm{H}_{6}}
\end{aligned}
$$

with $c_{5}$ denoting the recombination rate and $c_{6}$ the dissociation rate. The solution for (5) is

$$
\begin{aligned}
& \theta_{\mathrm{C}_{2} \mathrm{H}_{6}}(t) \\
= & {\left[\alpha\left(\frac{2}{c_{6}-\left(c_{1}+c_{4}\right)}-\frac{1}{c_{6}-2 c_{1}}-\frac{1}{c_{6}-2 c_{4}}\right) \mathrm{e}^{-c_{6} t}\right.} \\
& \left.+\frac{1}{c_{6}-2 c_{1}} \mathrm{e}^{-2 c_{1} t}+\frac{1}{c_{6}-2 c_{4}} \mathrm{e}^{-2 c_{4} t}-\frac{2}{c_{6}-\left(c_{1}+c_{4}\right)} \mathrm{e}^{-\left(c_{1}+c_{4}\right) t}\right]
\end{aligned}
$$

However, if the recombination process occurs during thermal heating (in the solid and before desorption) then the probability of formation of $\mathrm{C}_{2} \mathrm{H}_{6}, P$, is simply proportional to the square of $\theta_{\mathrm{CH}_{3}}(T)$ with $T$ indicating the irradiation time $T$

$$
P_{\mathrm{C}_{2} \mathrm{H}_{6}}(T)=\alpha_{1}\left(\mathrm{e}^{-2 c_{1} T}+\mathrm{e}^{-2 c_{4} T}-2 \mathrm{e}^{-\left(c_{1}+c_{4}\right) T}\right)
$$

As shown in Figure 3, fitting to the total intensity of $\mathrm{C}_{2} \mathrm{H}_{6}$ produced in the solid, sum of all its detected fragments, as a function of electron irradiation time $T$ yields $c_{1}^{-1} \sim 1 \times 10^{3} \mathrm{~s}$ and $c_{4}^{-1} \sim 1 \times 10^{6}$.

An independent evaluation of the rate of electron induced dehydrogenation of a methane molecule in a solid matrix $A$ can be made as follows: the reaction depth $d=\lambda=$ $300 \mathrm{n} A ; P_{1}=1.13 \times 10^{-5}$, the probability that a $\mathrm{CH}_{4}$ molecule to be dissociated in a second is $P_{1} \cdot P_{2} \cdot c_{\mathrm{CH}_{4}}=$ $9.1 \times 10^{-7} c_{\mathrm{CH}_{4}}$. We may reasonable assume the total number of hits to be at least 100 with a similar s, $c_{\mathrm{CH}_{4}}=0.8$ then the real probability of dissociating a $\mathrm{CH}_{4}$ molecule can be $7.3 \times 10^{-5}$ or more or a constant rate of $1.4 \times 10^{4} \mathrm{~s}$.

This estimate is just about a factor of 14 off the experimental value of $A=1 \times 10^{3} \mathrm{~s}$. Considering the roughness of this simple model with large uncertainties in the parameters used such as $\lambda$ and the average hits per incident electron, and of the assumption of a uniform distribution of electron- $\mathrm{CH}_{4}$ collision probability over the depth, we may certainly regard the experimentally determined value of $A$ reasonable. We also point out that in 100 hits only $8 \%$ will produce dissociation with a total energy loss of only $40 \mathrm{eV}$ or something more.

Further, by assuming that all $\mathrm{CH}$ is produced in the solid via dissociation of $\mathrm{CH}_{4}$ just as in the case of $\mathrm{CH}_{3}$, we fitted the intensity of $\mathrm{C}_{2} \mathrm{H}_{2}$ after subtracting the cracking fraction of $\mathrm{C}_{2} \mathrm{H}_{6}$ with use of Equation (5) to (lower panel of Figure 3). The so determined time constant $A=2 \times 10^{3}$ s is quite similar to the value obtained 
for $\mathrm{C}_{2} \mathrm{H}_{6}$, though the assumed hypothesis is quite rough.

\subsection{Monte Carlo Simulation}

For a better knowledge of our experimental results we also developed a simple Monte Carlo code which simulates the irradiation of a solid methane sample with electrons. The code follows the basic ideas of the program CASINO.

CASINO (monte CArlo SImulation of electroNs in sOlids) has been programmed by the research team of Raynald Gauvin and it is well described in Drouin et al. [19]. Essentially the main part of the Monte Carlo program is the simulation of a complete electron trajectory. The electron lands randomly on the sample with an initial penetration angle fixed by the user. The distance between two successive collisions is evaluated using the equations

$$
\begin{gathered}
L=-\lambda_{e l} \log (R)[\mathrm{nm}] \\
\frac{1}{\lambda_{e l}}=\rho N_{o} \sum_{i=1}^{n} \frac{C_{i} \sigma_{e l}^{i}}{A_{i}}
\end{gathered}
$$

where $C_{i}$ and $A_{i}$ are the weight fraction and atomic weight of element $i, \rho$ is the density of the region $\left(\mathrm{g} / \mathrm{cm}^{3}\right)$, $N_{o}$ is the Avogrado's constant and $\sigma_{i e l}$ is the electron total cross section.

The energy between collisions can be calculated as

$$
E_{i+1}=E_{i}+\frac{\mathrm{d} E}{\mathrm{~d} S} L
$$

with $\frac{\mathrm{d} E}{\mathrm{~d} S}$ given in Drouin et al. [19]. The scattering angle is determined as described in Hovington et al. [20]. The steps given above are repeated until the electron energy is less than a predetermined value or the electron escapes from the sample.

Our code, MethDiss (Methane Dissociation), will be described in depth in a separate paper. Here we briefly report that we consider a solid $\mathrm{CH}_{4}$ sample with dimensions of $(300 \times 300 \times 300) \mathrm{nm}^{3}$. A layer of $\mathrm{CH}_{4}$ molecules has a depth of $3 \mathrm{~nm}$, the outer layer is on the $\mathrm{xy}$ plane with $z=0$, the inner layers have $z<0$. Electrons with a given energy come from $z>0$ in direction perpendicular to the sample with a flux rescaled by the experimental situation. Electrons path in the solid is followed by using the steps given above. At each collision we considered the possibility that the molecule hit by the electron is dissociated or ionized. Cross sections (total, dissociation, ionization) for electron collisions with hydrocarbons are taken from Shirai et al. [15]. Upon electron irradiation the sample, initially formed by $\mathrm{CH} 4$ molecules only, becomes populated by $\mathrm{CH}_{\mathrm{x}}$ and $\mathrm{C}_{2} \mathrm{H}_{\mathrm{x}}$ hydrocarbons. We assumed that formation of more complex hydrocarbons can take place when two nearest neighbour molecules react together. With these assumptions we obtained, in our simulations, the formation of all the molecules detected during the experiments. Simulations and data analysis of the most comprehensive model are not completed yet. We took into account dissociation of $\mathrm{CH}_{\mathrm{x}}$ and $\mathrm{C}_{2} \mathrm{H}_{\mathrm{x}}$ hydrocarbons (which brings to the production of neutral and ionized species) and chemical reactions between them, $\mathrm{H}_{2}$ production and diffusion, $\mathrm{H}$ recombination, neutralization of ionized species due to low energy electrons. Our goal is to be able to fit the concentrations of all the detected species as a function of the irradiation time. Here we give the results of a preliminary, very simple, simulation. We considered only dissociation of $\mathrm{CH}_{4}$ molecules in $\mathrm{CH}_{3}$ and $\mathrm{H}$. In Figure 4 we show the comparison between normalized experimental data (black squares) and simulation (black line) for $\mathrm{C}_{2} \mathrm{H}_{6}$ formation. So, using the simulated irradiation time as the only free parameter our simulation is able to reproduce experimental data.

\section{Conclusion}

In this work we presented an experimental and theoretical investigation on $500-3000 \mathrm{eV}$ electron induced chemical reactions in solid methane at $20 \mathrm{~K}$. Our study confirms and improves that one of Bennet et al. [3]. We

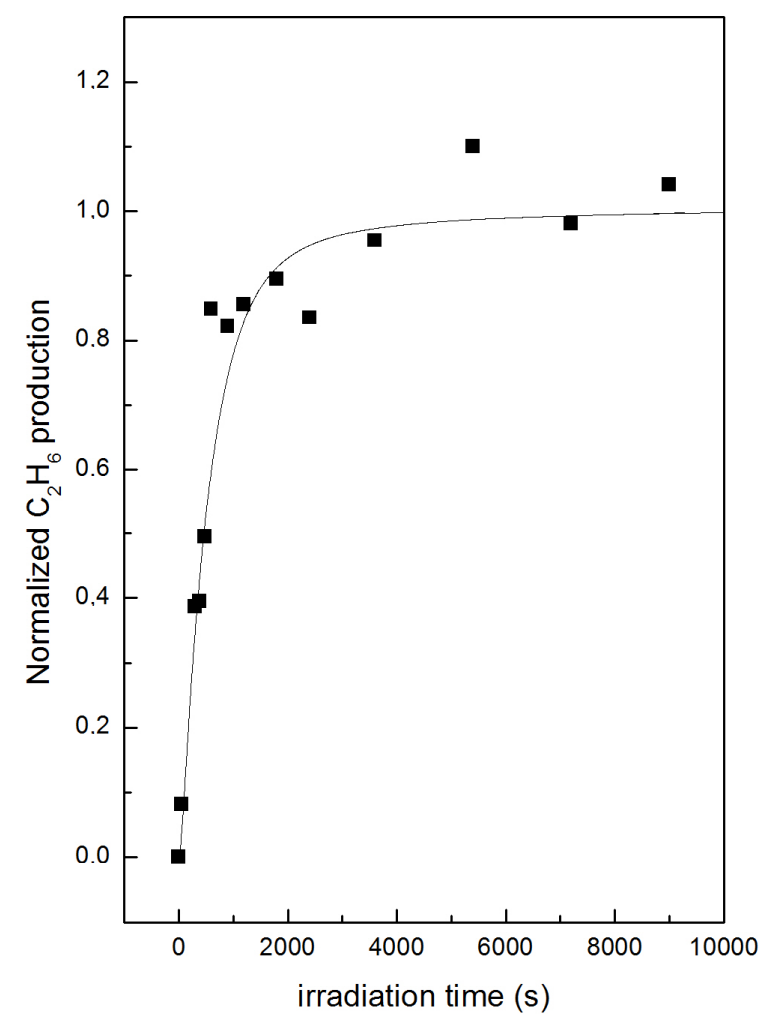

Figure 4. Total $\mathrm{C}_{2} \mathrm{H}_{6}$ (a) intensity produced by $2 \mathrm{keV}$ electron bombardment on a thick solid $\mathrm{CH}_{4}$ film as a function of irradiation time. The continuous curve is obtained using the CASINO results (see text). 
obtain that the primary effects of irradiations is the formations of $\mathrm{CH}_{3}, \mathrm{CH}_{2}$ and $\mathrm{CH}$ fragments from the dehydrogenation of methane. The formations of ethane and acetylene is obtained, during solid ice irradiation, from a simple recombination reaction between two adjacent $\mathrm{CH}_{3}$ or $\mathrm{CH}$ radicals. Our experimental results are confirmed with the implementation of a theoretical model and a Monte Carlo simulation that discussed the ethane (acetylene) formation in terms of association of two adjacent methyl radicals $\mathrm{CH}_{3}\left(\mathrm{CH}_{2}\right)$ during electron irradiation. At this stage we assume that the effect of thermal desorption is negligible, but more efforts are necessary to introduce the effects of thermal desorption on dicarbonic species formation.

\section{REFERENCES}

[1] E. Garand and P. A. Rowntree, "The Mechanism of Hydrogen Formation Induced by Low-Energy Electron Irradiation of Hexadecanethiol Self-Assembled Monolayers," The Journal of Physical Chemistry B, Vol. 109, No. 26, 2005, pp. 12927-12934. doi:10.1021/jp050817k

[2] H. F. Winters, "Dissociation of Ethane by Electron Impact," Chemical Physics, Vol. 36, No. 3, 1979, pp. 353364. doi:10.1016/0301-0104(79)85019-3

[3] C. J. Bennet, C. S. Jamieson, Y. Osamura and R. I. Kaiser, "Laboratory Studies on the Irradiation of Methane in Inter- stellar, Cometary, and Solar System Ices," The Astrophy- sical Journal, Vol. 653, No. 1, 2006, p. 792. doi:10.1086/508561

[4] Scheffler and Elsasser, "Physics of the Galaxy and Interstellar Matter," Springer, Berlin, 1988.

[5] D. C. B. Whittet, "Observations of Molecular Ices," In: T. J. Millar and D. A. Williams, Eds., Dust and Chemistry in Astronomy, Cambridge University Press, Cambridge, 1993, p. 201.

[6] Hollenbach and Thronson, "Interstellar Processes," Proceedings of the Symposium on Interstellar Processes, Grand Teton National Park, 1-7 July 1986, Vol. 134.

[7] W. A. Schutte, "Production of Organic Molecules in Interstellar Ices," Advances in Space Research, Vol. 30, No. 6, 2002, pp. 1409-1417. doi:10.1016/S0273-1177(02)00500-8

[8] R. I. Kaiser and K. Roessler, "Theoretical and Laboratory Studies on the Interaction of Cosmic-Ray Particles with Interstellar Ices. III. Suprathermal Chemistry-Induced Formation of Hydrocarbon Molecules in Solid Methane $\left(\mathrm{CH}_{4}\right)$, Ethylene $\left(\mathrm{C}_{2} \mathrm{H}_{4}\right)$, and Acetylene $\left(\mathrm{C}_{2} \mathrm{H}_{2}\right)$," The As- trophysical Journal, Vol. 503, No. 2, 1998, p. 959. doi: $10.1086 / 306001$

[9] A. Wada, N. Mochizuki and K. Hiraoka, "Methanol Formation from Electron-Irradiated Mixed $\mathrm{H}_{2} \mathrm{O} / \mathrm{CH}_{4}$ Ice at 10 K," The Astrophysical Journal, Vol. 644, No. 1, 2006, p. 300. doi:10.1086/503380

[10] Zheng, Jewitt, Osamura and Kaiser, "Formation of Nitrogen and Hydrogen-bearing Molecules in Solid Ammonia and Implications for Solar System and Interstellar Ices," The Astrophysical Journal, Vol. 674, No. 2, 2008, p. 1242. doi: $10.1086 / 523783$

[11] P. Ehrenfreund, S. B. Charneley and D. Wooden, "Comets II," University of Arizona Press, Tucson, 2004.

[12] T. Y. Brooke, A. T. Tokunaga, H. A. Weaver, J. Crovisier, D. Bockelée-Morvan and D. Crisp, "Detection of Acetylene in the Infrared Spectrum of Comet Hyakutak," $\mathrm{Na}$ ture, Vol. 383, 1996, pp. 606-608. doi: $10.1038 / 383606 \mathrm{a} 0$

[13] http://www.sisweb.com/referenc/source/exactmas.htm

[14] M. Barberio, P. Barone, R. Vasta, G. Manicò and F. Xu, "Formation of Molecular Nitrogen and Diazene by Electron Irradiation of Solid Ammonia," Thin Solid Film, Vol. 520, No. 16, 2012, pp. 5479-5481. doi:10.1016/j.tsf.2012.03.112

[15] T. Shirai, et al., "Analytic Cross Sections for Electron Collisions with Hydrocarbons: $\mathrm{CH}_{4}, \mathrm{C}_{2} \mathrm{H}_{6}, \mathrm{C}_{2} \mathrm{H}_{4}, \mathrm{C}_{2} \mathrm{H}_{2}$, $\mathrm{C}_{3} \mathrm{H}_{8}$, and $\mathrm{C}_{3} \mathrm{H}_{6}$," Atomic Data and Nuclear Data Tables, Vol. 80, No. 2, 2002, pp. 147-204. doi:10.1006/adnd.2001.0878

[16] D. J. Alberas-Sloan and J. M. White, "Low-Energy Electron Irradiation of Methane on $\operatorname{Pt}(111)$," Surface Science, Vol. 365 No. 2, 1996, pp. 212-228. doi:10.1016/0039-6028(96)00720-0

[17] http://xdb.lbl.gov/Section3/Sec_3-2.html

[18] M. A. Huels, P. C. Dugal and L. Sanche, "Degradation of Functionalized Alkanethiolate Monolayers by $0-18 \mathrm{eV}$ Electrons," Journal of Chemical Physics, Vol. 118, No. 24, 2003, pp. 11168-11179. doi:10.1063/1.1574791

[19] D. Drouin, A. R. Couture, D. Joly, X. Tastet, V. Aimez and R. Gauvin, "CASINO V2.42-A Fast and Easy-toUse Modeling Tool for Scanning Electron Microscopy and Microanalysis Users," Scanning, Vol. 29, No. 3, 2007, p. 92.

[20] P. Hovington, D. Drouin and R. Gauvin, "CASINO: A New Monte Carlo Code in C Language for Electron Beam intEraction-Part I: Description of the Program," Scanning, Vol. 19, No. 1, 1997, pp. 1-14. doi:10.1002/sca.4950190101 